\title{
Incidence and prognostic factors in severe drug-induced interstitial lung disease caused by antineoplastic drug therapy in the real world
}

\author{
Sawako Kaku ${ }^{1,2} \cdot$ Hidehito Horinouchi $^{3}\left(\mathbb{D}^{\circ} \cdot\right.$ Hirokazu Watanabe $^{1} \cdot$ Kan Yonemori $^{4} \cdot$ Takuji Okusaka $^{5} \cdot$ Narikazu Boku $^{6}$. \\ Naoya Yamazaki ${ }^{7} \cdot$ Akira Kawai $^{8} \cdot$ Yuichiro Ohe $^{2,3} \cdot$ Masahiko Kusumoto $^{1}$
}

Received: 31 October 2021 / Accepted: 23 January 2022 / Published online: 7 February 2022

(c) The Author(s) 2022, corrected publication 2022

\begin{abstract}
Purpose Investigate the frequency and prognostic factors of severe drug-induced interstitial lung disease (DILD) caused by antineoplastic drugs regardless of cancer types or type of drugs.

Methods From 2014 to 2018, we reviewed patients with a history of antineoplastic agents administration in the real-world database of our hospital's electronic medical record and extracted patients who experienced "severe" DILD, requiring hospitalization with treatment or developed during hospitalization and required treatment. We collected patients' backgrounds, clinical and radiological features, laboratory data, treatment, and survival outcomes.

Results 19,132 cancer patients received antineoplastic drug therapy during the study period, and $120(0.62 \%)$ experienced severe DILD. The incidence of severe DILD in patients with thoracic cancer was highest among the patients included in this analysis ( $2.52 \%$ vs. $0.34 \%$ other cancers). Diffuse alveolar damage (DAD) pattern on CT was associated with higher mortality in patients with severe DILD compared with non-DAD pattern (hazard ratio [HR], 11.24; 95\% CI, 4.82-26.2). Multivariate analysis revealed that the DAD pattern at diagnosis as severe DILD (HR, 3.59; 95\% CI, 1.17-11.03), concurrent/previous interstitial lung disease (HR, 3.20; 95\% CI, 1.27-8.10), and ECOG performance status of 2-4 (HR, 3.81; 95\% CI, 1.10-13.17) were independent risk factors for mortality in patients with severe DILD.

Conclusions The frequency of severe DILD was highest in patients with thoracic cancer. The DAD pattern was associated with a poor outcome. From the perspective of DILD, special attention should be paid when administering antineoplastic agents to patients with thoracic cancer.
\end{abstract}

Keywords Drug-induced interstitial lung disease $\cdot$ Cancer $\cdot$ Thoracic cancer $\cdot$ Lung cancer $\cdot$ Malignant melanoma . Antineoplastic drug therapy $\cdot$ Chemotherapy $\cdot$ Molecular targeted drug therapy $\cdot$ Immune-checkpoint inhibitor $\cdot$ Diffuse alveolar damage

Hidehito Horinouchi

hhorinou@ncc.go.jp

1 Department of Diagnostic Radiology, National Cancer Center Hospital, 5-1-1 Tsukiji, Chuo-ku, Tokyo 104-0045, Japan

2 Course of Advanced Clinical Research of Cancer, Juntendo University Graduate School of Medicine, Tokyo, Japan

3 Department of Thoracic Oncology, National Cancer Center Hospital, 5-1-1 Tsukiji, Chuo-ku, Tokyo 104-0045, Japan

4 Department of Medical Oncology, National Cancer Center Hospital, 5-1-1 Tsukiji, Chuo-ku, Tokyo 104-0045, Japan
5 Department of Hepatobiliary and Pancreatic Oncology, National Cancer Center Hospital, 5-1-1 Tsukiji, Chuo-ku, Tokyo 104-0045, Japan

6 Department of Gastrointestinal Medical Oncology, National Cancer Center Hospital, 5-1-1 Tsukiji, Chuo-ku, Tokyo 104-0045, Japan

7 Department of Dermatologic Oncology, National Cancer Center Hospital, 5-1-1 Tsukiji, Chuo-ku, Tokyo 104-0045, Japan

8 Department of Musculoskeletal Oncology, National Cancer Center Hospital, 5-1-1 Tsukiji, Chuo-ku, Tokyo 104-0045, Japan 


\section{Introduction}

Drug-induced interstitial lung disease (DILD), also known as drug-induced pneumonitis or drug-induced pulmonary toxicity, is a significant treatment-related complication in cancer treatment (Sakurada et al. 2015; Leger et al. 2017). DILD is known to have substantial adverse clinical consequences in severe cases, including treatment-related death (Ando et al. 2006). A population-based study reported an incidence of respiratory failure attributable to druginduced interstitial lung disease of 6.6 per 100,000 patientyears, with more than half of the cases associated with chemotherapeutic agents (Dhokarh et al. 2012).

Many clinical trials and postmarketing surveillance referred to DILD as a potential adverse event or side effect and have examined both pathogenic and prognostic factors (Gemma et al. 2020; Osawa et al. 2015; Abdel-Rahman and Elhalawani 2015). Additionally, the use of drugs with new action mechanisms, such as molecular targeting agents and immune checkpoint inhibitors (ICIs), has increased the frequency of DILD (Johkoh et al. 2021). Among cancer-specific reports, chemotherapy-related pneumonitis reportedly occurs in approximately $30 \%$ of lung cancer patients and is believed to be the most common cause of treatment-related death (Minami-Shimmyo et al. 2012).

However, no real-world studies have investigated cross-sectional incidences and prognostic factors in druginduced interstitial lung disease regardless of cancer or drug. Additionally, the number of severe drug-induced interstitial lung disease cases is small, making it challenging to collect data. Thus, we investigated the incidence, mortality, and prognostic factors of severe DILD in cancer treatment, regardless of drug class or tumor type.

\section{Materials and methods}

\section{Patient selection and data collection}

We reviewed the medical records of patients who received antineoplastic drug therapies and extracted patients experiencing severe DILD retrospectively. To identify the incidence of severe DILD, we first collected data from all the patients who received antineoplastic drug therapy at our hospital during the study period. We extracted 19,132 patients with a history of antineoplastic drug administration in the real-world database of our hospital's electronic medical record. These data included patient age, sex, type of cancer, and the antineoplastic drugs administered. We then reviewed patients whose CT scan reports contained drug-induced lung injury, interstitial pneumonia, or similar terms during the study period. Next, we identified patients with newly appearing interstitial findings in the lungs, including drug-induced interstitial pneumonitis. Finally, we selected patients with "severe" drug-induced lung injury according to selection/exclusion criteria. The selection criteria for "severe" DILD were as follows: (1) patients receiving antineoplastic drugs, (2) patients repeating regular thoracic CT scans, (3) patients developing drug-induced interstitial lung disease because of antineoplastic drugs required hospitalization, and (4) patients developing drug-induced interstitial lung disease because of antineoplastic drugs during hospitalization.

The exclusion criteria for severe DILD were as follows: (1) patients with DILD caused by investigational drugs, (2) patients with DILD developed by treatments other than antineoplastic drugs, (3) patients who developed DILD because of antineoplastic drugs and hospitalized for observation, and (4) patients who developed DILD because of antineoplastic drugs during hospitalization without additional treatment. In evaluating DILD imaging patterns, two board-certified radiologists (M.K. and H.W.) independently reviewed chest computed tomography (CT) images obtained at the time of severe DILD and classified according to CT image pattern definitions ${ }^{5}$. The imaging patterns of ILD are as follows: (1) diffuse alveolar damage (DAD) pattern; (2) hypersensitivity pneumonia (HP) pattern; (3) organizing pneumonia (OP) pattern; and (4) others. In addition, we collected the data of patients' backgrounds, types of antineoplastic drugs administered, and other clinical information, including outcomes.

\section{Statistical analysis}

Survival time was defined as the period from the onset of severe DILD until death or censoring. Cancer-specific death was defined as death after recovery from severe DILD. As for the overall survival analysis, we used the Kaplan-Meyer estimate and the Cox proportional hazard model. We considered cancer-related death as a competing risk for DILDrelated death in this analysis. The cumulative incidence of DILD-related deaths was calculated using a cumulative incidence function, taking into account competing risks. The Fine-Gray hazard model was used to identify prognostic factors of severe DILD to adjust for the competing risk (Fine and Gray 1999). The factors used in the univariate and multivariate analyses were as follows: the type of cancer, history of thoracic surgery, smoking history, history of irradiation to the chest, concurrent/previous interstitial lung disease including drug-induced interstitial lung disease, ECOG performance status (PS) on admission, oxygen requirement on admission, the existence of emphysema, patterns of interstitial changes in the lungs (DAD pattern, OP pattern, HP pattern, and others), and use of steroid pulse therapy. 
Statistical significance was set at $P<0.05$. All statistical analyses were performed using EZR version 1.53 (Saitama Medical Center, Jichi Medical University, Saitama, Japan) and a graphical user interface (R Foundation for Statistical Computing, Vienna, Austria).

\section{Results}

\section{The incidence and patient characteristics of severe DILD}

From 2014 to 2018, a total of 19,132 patients were treated with antineoplastic drugs at our institution; of these patients, $120(0.62 \%)$ developed severe DILD (Fig. 1).

The incidence of severe DILD among all patients who received antineoplastic drugs was highest for patients with thoracic cancer $(2.52 \%)$, taking the frequency of each type of cancer into consideration. Skin malignancy $(1.53 \%)$ and hepatobiliary cancer $(0.70 \%)$ had the second and third

Fig. 1 Patient flow diagram

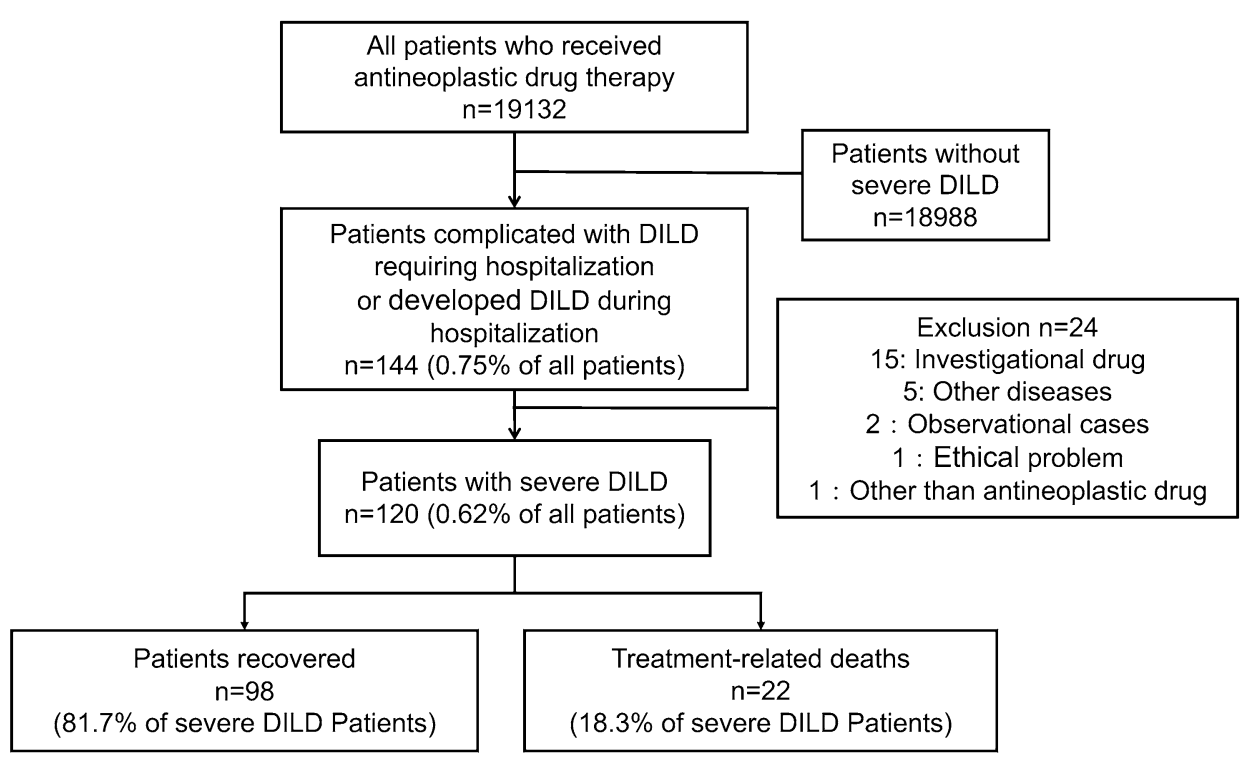

Fig. 2 The incidence rate of severe DILD and the number of patients who received antineoplastic drug therapy

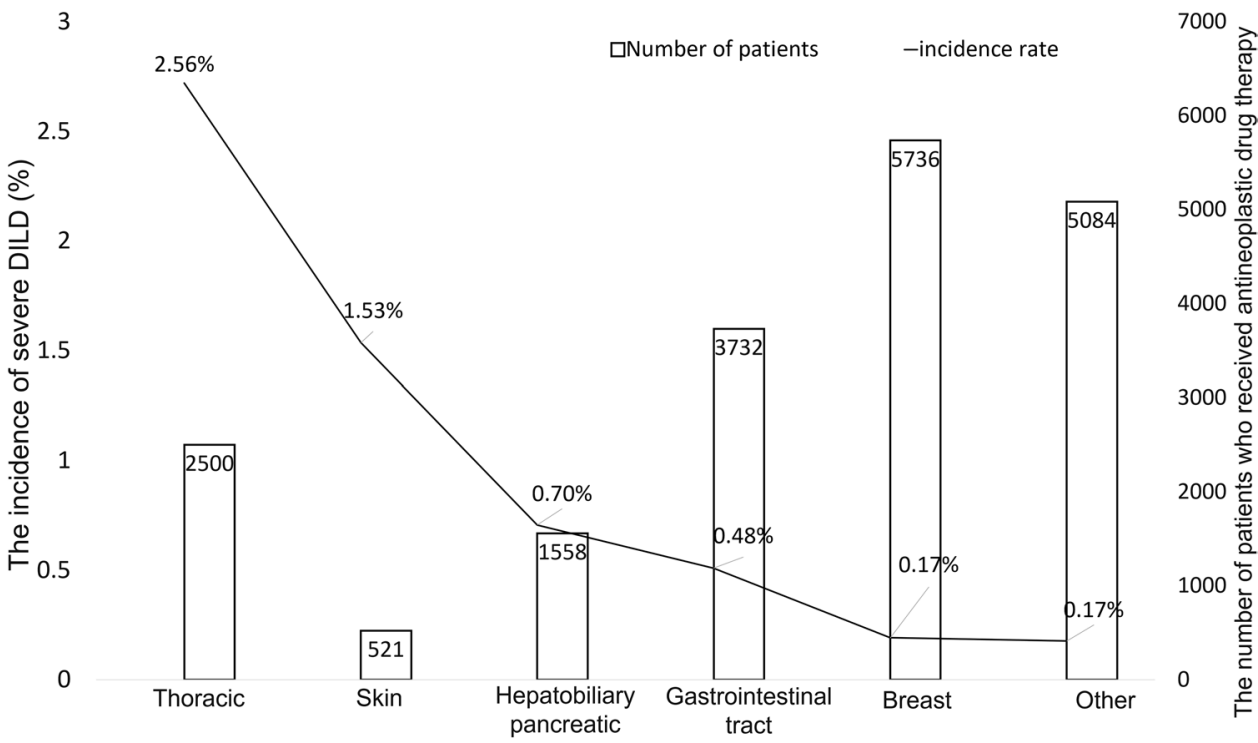


Table 1 Patient characteristics

\begin{tabular}{lll}
\hline Patient backgrounds & $N=120$ & \\
\hline Age mean (range) & 66 & $(25-86)$ \\
Sex & $N$ & $\%$ \\
Male & 84 & 70.00 \\
Female & 36 & 30.00 \\
Etiology of cancer & & \\
Lung & 64 & 53.30 \\
Biliary tract and pancreas & 11 & 9.20 \\
Breast & 10 & 8.30 \\
Upper GI & & 8.30 \\
Lower GI & 10 & 6.70 \\
Melanoma & 8 & 6.70 \\
Other & 8 & 7.50 \\
Performance status & 9 & \\
0 & & 5.10 \\
1 & 6 & 55.10 \\
2 & 65 & 22.90 \\
3 & 27 & 11.90 \\
4 & 14 & 5.00 \\
Unknown & 6 & 1.60 \\
Number of regimen (mean, range) & $3.0[1.00-9.00]$ \\
Concurrent/previous ILD & \\
Smoking history & 15 & 12.50 \\
Operation history & 62 & 53.30 \\
Radiation history & 24 & 20.00 \\
Oxygen supplementation & 18 & 15.00 \\
Steroid pulse therapy & 91 & 75.83 \\
\hline & 60 & 50.00 \\
\hline
\end{tabular}

${ }^{\mathrm{a}} G I$ gastrointestinal

${ }^{\mathrm{b}} I L D$ interstitial lung disease

highest incidences of severe DILD following thoracic cancer (Fig. 2). The characteristics of the patients with severe DILD are shown in Table 1 . The median age was 66 years (range: $25-86$ years), and the proportion of males was higher than that of females $(70.0 \%$ vs. $30.0 \%)$. Thoracic cancer accounted for more than half of the primary organs (53.3\%). The following most common sites of primary cancer were hepatobiliary and pancreatic cancer (9.2\%), breast cancer and upper gastrointestinal cancer (8.3\%), lower gastrointestinal cancer, and melanoma $(6.7 \%)$. The majority of the patients' PS scores were $1(55.1 \%)$ or 2 (22.9\%). Among patients who developed severe DILD, 15 $(12.5 \%)$ had concurrent/previous interstitial lung disease, $62(53.3 \%)$ had a smoking history, and $18(15.0 \%)$ had a history of thoracic radiotherapy. In addition, 91 (75.8\%) required oxygen supplements, and $60(50.0 \%)$ were treated by steroid pulse therapy. The data cut-off was March 31, 2019. The median follow-up time from the onset of severe DILD was 134.5 days (range: 1-1229 days).

\section{The outcome of severe DILD caused by antineoplastic drugs}

The mortality rate in all 126 patients who developed severe DILD was $18.3 \%(n=23)$ (Table 2). 98 patients recovered, and over half of them (99 patients: $74.2 \%$ ) required the continued use of corticosteroids to maintain the resolution of DILD. Concerning the type of cancer treatment, the highest mortality rate was observed among cases of severe DILD caused by conventional cytotoxic agents. In detail, the proportions of fatal outcomes were as follows: cytotoxic agent, $21.1 \%$ (16/76); molecularly targeted agent, 9.5\% (2/21); and ICIs, $17.4 \%$ (4/23). Patients receiving ICIs were the least likely to achieve steroid withdrawal after the onset of severe DILD (13.0\%). All the severe DILD-related deaths arising from the use of molecularly targeted agents occurred in patients receiving epidermal growth factor receptor (EGFR) tyrosine kinase inhibitors (TKIs). Among the severe DILD patients receiving PD-1 inhibitors, 3 out of 23 patients (13.0\%) died. Of these 3 deaths, 1 patient had received combination therapy with a PD-1 inhibitor and CTLA-4 inhibitor. In contrast, among the 65 patients who received PD-L1 inhibitors and developed severe DILD, only one patient (1.54\%) died.

\section{CT pattern of severe DILD caused by antineoplastic drugs}

The most common CT pattern in patients with severe DILD was the HP pattern (39.2\%). The OP pattern (29.1\%) and the DAD pattern $(12.5 \%)$ were the second and third most common. The mortality rate of patients with severe DILD was highest for those with the DAD pattern (53.3\%), followed by those with an OP pattern (11.4\%) and those with an HP pattern (6.4\%). The proportion of patients who could discontinue corticosteroids after severe DILD was inversely associated with the mortality rate (Table 3). The HP pattern was commonly observed in patients who had received gemcitabine, docetaxel, nivolumab, and irinotecan. The severe DILD patients with an HP pattern had the highest steroid withdrawal rate $(31.9 \%)$. The OP pattern of severe DILD was frequent among patients treated by pembrolizumab and nivolumab. Paclitaxel was the most common drug suspected of causing the DAD pattern in patients with severe DILD, and none of these patients were able to withdraw from steroid treatment.

\section{Cumulative incidences and mortality of DILD}

In Fig. 3a, the cumulative incidence of severe DILDrelated deaths, taking cancer-related deaths into account as a competing risk, was shown. The mortality rate was higher in the group with the DAD pattern at the onset of 
Table2 Incidence and outcome of severe DILD caused by antineoplastic drugs

\begin{tabular}{|c|c|c|c|c|c|c|c|c|c|c|}
\hline & \multirow[t]{2}{*}{$\begin{array}{l}\text { Number of } \\
\text { patients }\end{array}$} & \multirow[t]{2}{*}{ Number of drugs } & \multicolumn{2}{|c|}{$\begin{array}{l}\text { Severe DILD } \\
\text { with hospitali- } \\
\text { zation }\end{array}$} & \multicolumn{2}{|c|}{$\begin{array}{l}\text { Imaging pattern } \\
\text { of DILD }\end{array}$} & \multicolumn{2}{|c|}{$\begin{array}{l}\text { Successful ster- } \\
\text { oid withdrawal }\end{array}$} & \multicolumn{2}{|c|}{$\begin{array}{l}\text { Number of } \\
\text { treatment- } \\
\text { related death in } \\
\text { severe DILD }\end{array}$} \\
\hline & & & $N$ & $\%$ & & & $N$ & $\%$ & $N$ & $\%$ \\
\hline Total & 19,132 & 56,651 & 120 & 0.62 & & & 31 & 25.83 & 22 & 18.33 \\
\hline \multirow[t]{4}{*}{ Cytotoxic agents } & 17,251 & 51,083 & 76 & 0.44 & $\mathrm{HP}$ & 34 & 23 & 30.26 & 16 & 21.05 \\
\hline & & & & & DAD & 13 & & & & \\
\hline & & & & & OP & 12 & & & & \\
\hline & & & & & other & 17 & & & & \\
\hline \multirow[t]{5}{*}{ Molecular targeted agents } & 3989 & 5385 & 21 & 0.52 & HP & 5 & 6 & 28.57 & 2 & 9.52 \\
\hline & & & & & DAD & 1 & & & & \\
\hline & & & & & $\mathrm{OP}$ & 12 & & & & \\
\hline & & & & & Other & 2 & & & & \\
\hline & & & & & no CT & 1 & & & & \\
\hline \multirow[t]{5}{*}{ Immune-checkpoint inhibitors } & 975 & 1165 & 23 & 2.35 & HP & 8 & 3 & 13.04 & 4 & 17.39 \\
\hline & & & & & DAD & 1 & & & & \\
\hline & & & & & $\mathrm{OP}$ & 11 & & & & \\
\hline & & & & & Other & 2 & & & & \\
\hline & & & & & no CT & 1 & & & & \\
\hline
\end{tabular}

Table 3 CT pattern of severe DILD caused by antineoplastic drugs

\begin{tabular}{|c|c|c|c|c|c|c|c|}
\hline \multirow{2}{*}{$\begin{array}{l}\text { Imaging pattern } \\
\text { of DILD } \\
\\
\text { HP }\end{array}$} & \multirow{2}{*}{$\begin{array}{l}N \\
\quad \\
\\
\\
\\
\\
47 \\
\end{array}$} & \multirow{2}{*}{$\begin{array}{l}\% \\
39.17 \\
\end{array}$} & \multirow[t]{2}{*}{ Suspected drug } & \multirow[t]{2}{*}{$N$} & \multicolumn{2}{|c|}{$\begin{array}{l}\text { Number of } \\
\text { treatment-related } \\
\text { deaths and } \\
\text { mortality (\%) } \\
\text { in patients with } \\
\text { severe DILD }\end{array}$} & \multirow{2}{*}{$\begin{array}{l}\text { Successful steroid } \\
\text { withdrawal }(\%) \\
15(31.91)\end{array}$} \\
\hline & & & & & 3 & $(6.38)$ & \\
\hline & & & Gemcitabine & 8 & 1 & (11.11) & \\
\hline & & & Paclitaxel & 7 & 0 & $(0.00)$ & \\
\hline & & & Nivolumab & 6 & 0 & $(0.00)$ & \\
\hline & & & Irinotecan & 4 & 0 & $(0.00)$ & \\
\hline & & & Docetaxel & 3 & 0 & $(0.00)$ & \\
\hline & & & Osimertinib & 3 & 0 & $(0.00)$ & \\
\hline & & & Pembrolizumab & 3 & 0 & $(0.00)$ & \\
\hline & & & Other & 13 & 2 & (15.38) & \\
\hline \multirow[t]{6}{*}{$\mathrm{OP}$} & 35 & 29.17 & & & 4 & (11.43) & $9(25.71)$ \\
\hline & & & Pembrolizumab & 7 & 1 & (14.29) & \\
\hline & & & Nivolumab & 4 & 2 & $(50.00)$ & \\
\hline & & & Amrubicin & 3 & 0 & $(0.00)$ & \\
\hline & & & Afatinib & 3 & 0 & $(0.00)$ & \\
\hline & & & Other & 18 & 1 & $(5.56)$ & \\
\hline \multirow[t]{4}{*}{ DAD } & 15 & 12.50 & & & 8 & $(53.33)$ & $0(0.00)$ \\
\hline & & & Paclitaxel & 4 & 2 & $(50.00)$ & \\
\hline & & & Pemetrexed & 2 & 1 & $(50.00)$ & \\
\hline & & & Other & 9 & 5 & $(55.56)$ & \\
\hline Other & 21 & 17.50 & & 21 & 6 & $(28.57)$ & $7(33.33)$ \\
\hline No CT & 2 & 1.67 & & 2 & 1 & $(50.00)$ & $0(0.00)$ \\
\hline
\end{tabular}



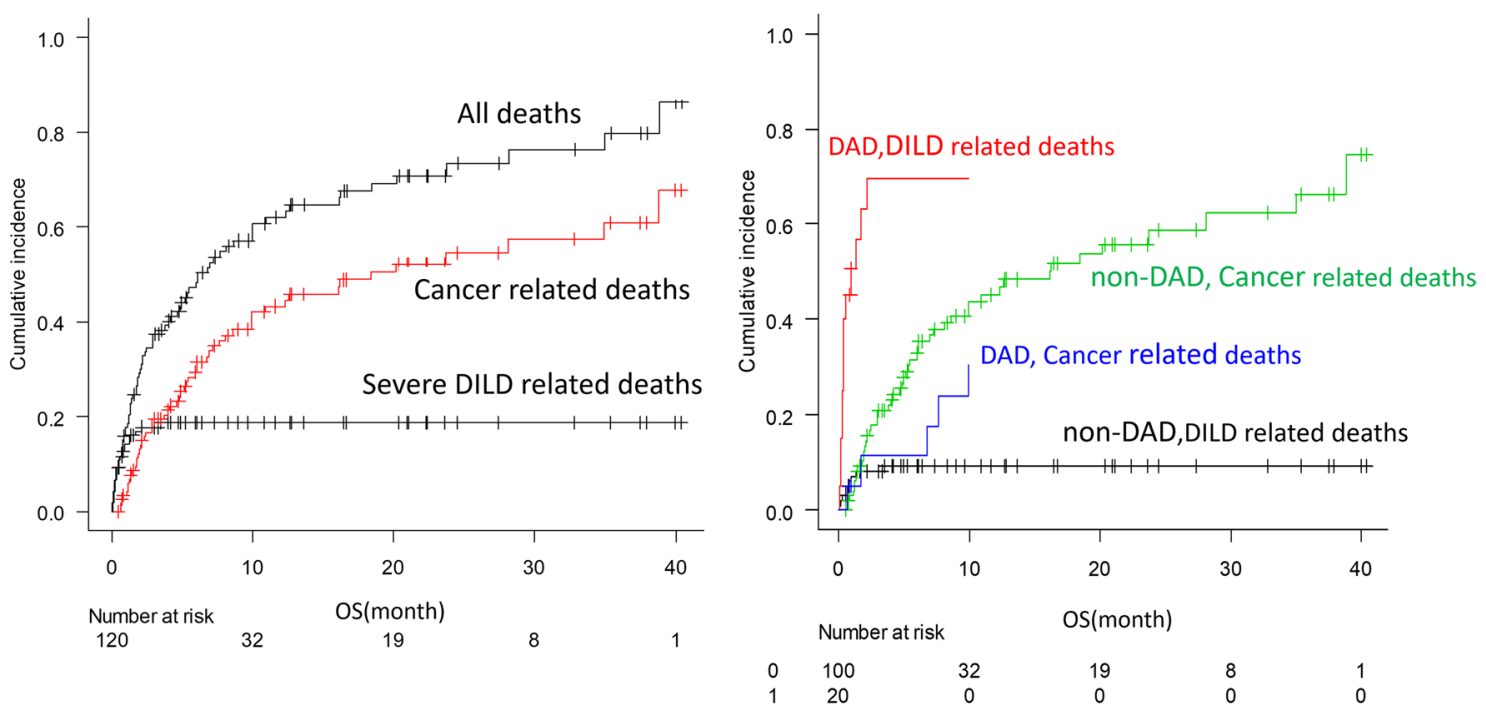

Fig. 3 a Cumulative incidence of severe DILD-related death, cancer death, and all death. b Cumulative incidence of DILD and cancer deaths classified by presence or absence of DAD pattern

severe DILD (DAD vs. non-DAD: $53.3 \%$ vs. $13.3 \%$ ). The DAD pattern was associated with increased mortality in patients with severe DILD (hazard ratio [HR], 11.24; 95\% CI, 4.82-26.2). The median survival time in patients with severe DILD with the DAD pattern was 10 days (range: 2-65 days) (Fig. 3b).

\section{Prognostic factors of severe DILD}

In univariate analyses, male sex, a history of thoracic surgery, a concurrent/previous interstitial lung disease, a history of smoking, ECOG PS of 2 or more, and the DAD pattern were associated with a higher risk of severe DILD-related death. Multivariate analysis showed that an ECOG PS of 2 or more (HR, 3.81; 95\% CI, 1.10-13.17), the DAD pattern (HR, 3.59; 95\% CI, 1.17-11.03), and concurrent/previous
Table 4 Univariate and multivariate analyses of prognostic factors of severe DILD

\begin{tabular}{|c|c|c|c|c|c|c|}
\hline \multirow[t]{2}{*}{ Factors } & \multicolumn{3}{|c|}{ Univariate analysis } & \multicolumn{3}{|c|}{ Multivariate analysis } \\
\hline & Hazard ratio & $95 \% \mathrm{CI}$ & $P$ value & Hazard ratio & $95 \% \mathrm{CI}$ & $P$ value \\
\hline \multicolumn{7}{|l|}{ Sex } \\
\hline Male & 1 & $0.01-0.73$ & 0.02 & 1 & $0.05-3.6$ & 0.42 \\
\hline Female & 0.09 & 0.44 & & & & \\
\hline \multicolumn{7}{|c|}{ History of lung surgery } \\
\hline Absence & 1 & $1.05-5.66$ & 0.04 & 1 & $0.59-5.21$ & 0.31 \\
\hline Presence & 2.44 & 1.76 & & & & \\
\hline \multicolumn{7}{|c|}{ History of interstitial lung disease } \\
\hline Absence & 1 & $2.24-12.67$ & $<0.01$ & 1 & $1.27-8.10$ & 0.01 \\
\hline Presence & 5.33 & 3.2 & & & & \\
\hline \multicolumn{7}{|c|}{ Smoking history } \\
\hline Absence & 1 & $1.18-22.63$ & 0.03 & 1 & $0.23-6.88$ & 0.78 \\
\hline Presence & 5.18 & 1.26 & & & & \\
\hline \multicolumn{7}{|c|}{ Performance status } \\
\hline $0-1$ & 1 & $2.32-16.46$ & $<0.01$ & 1 & $1.10-13.17$ & 0.03 \\
\hline$\geq 2$ & 6.18 & 3.81 & & & & \\
\hline \multicolumn{7}{|c|}{ DAD pattern on $\mathrm{CT}$} \\
\hline Absence & 1 & $4.82-26.20$ & $<0.01$ & 1 & $1.17-11.03$ & 0.02 \\
\hline Presence & 11.24 & 3.59 & & & & \\
\hline
\end{tabular}


interstitial lung disease (HR, 3.20; 95\% CI, 1.27-8.10) were independent prognostic factors (Table 4).

\section{Discussion}

To our knowledge, this is the first report to reveal the incidence and prognostic factor of severe DILD among the most significant number of patients who received antineoplastic drug therapy irrespective of cancer types or drug types. We examined 19,132 consecutive patients who received antineoplastic drug therapy and underwent regular thoracic CT examinations. In summary, thoracic cancer had the highest number $(n=64)$ and incidence rate $(2.56 \%)$ of severe DILD. The incidence and mortality of severe DILD varied among the types of antineoplastic drugs. In addition, the DAD pattern, concurrent/previous interstitial lung disease, and an ECOG PS of 2 or more were identified as independent prognostic factors of severe DILD. These findings help to predict treatment-related deaths caused by DILD regardless of the type of malignant neoplasm or administered antineoplastic drug therapy.

Our study highlighted that the incidence of severe DILD was highest among patients with thoracic cancer. The incidence of severe DILD in patients with thoracic cancer was more elevated than patients with other cancers $(2.5 \%$ vs. $0.3 \%)$. In the previous reports, the incidence of DILD during cancer drug therapy in non-small cell lung cancer was reported to be 5\% (Fujimoto et al. 2016). The incidence of drug-induced interstitial lung disease in thoracic cancer patients in our study was similar. This tendency was consistent throughout the types of antineoplastic drugs administered, such as cytotoxic agents, molecular target agents, and ICIs. Regarding the incidence and severity of DILD in cross-sectional analyses covering multiple types of cancers or treatments, a large retrospective cohort study examining gemcitabine-related DILD reported that patients with lung cancer were more likely to develop DILD than patients with other cancers (Hamada et al. 2016). Among patients receiving combination therapy consisting of docetaxel and gemcitabine, lung cancer patients also had a higher risk of developing severe DILD than breast cancer patients (Binder et al. 2011).

Additionally, severe DILD in patients with cancers other than thoracic cancer and skin malignancy were almost limited to patients treated with cytotoxic agents. Patients with NSCLC had a higher incidence of grade 3 or higher pneumonia than patients with melanoma $(1.8 \%$ vs. $0.2 \%$; $P<0.001$ ) (Nishino et al. 2016), while skin malignancies had the second-highest incidence of severe DILD, and all the patients with melanoma who developed DILD had been treated with ICI. In a meta-analysis of drug-induced interstitial lung disease with PD-1 inhibitors, the incidence of grade 3 pneumonia in patients treated with PD-1 inhibitors was $0.2 \%$. Patients with thoracic cancer are more susceptible to severe drug-induced interstitial lung disease because of the tumor burden and the presence of background lung injuries, such as interstitial lung abnormalities or emphysema (Bouros et al. 2002; Toh et al. 2004). Our results demonstrate the different backgrounds and susceptibilities of patients who develop severe DILD among patients with various tumors and treated with various antineoplastic drugs.

Concurrent/previous interstitial lung disease (ILD) and a PS of 2-4 were identified as predictors of a poor prognosis after the onset of severe DILD. Although these factors have been previously detected as risk factors for the development of DILD (Minami-Shimmyo et al. 2012; Hamada et al. 2016; Tirumani et al. 2015; Nakagawa et al. 2012), a few studies have examined them as prognostic factors of DILD. Preexisting ILD and a decreased PS have been identified as prognostic factors in postmarketing surveys of erlotinib and a meta-analysis of 24 phase III clinical trials of EGFRTKIs (Gemma et al. 2014; Qi et al. 2015). Our present study showed that pre-existing ILD and a decreased PS could be considered prognostic factors of severe DILD even when all antineoplastic drugs were comprehensively considered. We confirmed that these risk factors for developing DILD affect prognosis, since our study focused on severe cases, and our findings underscore the importance of these factors. Given the potentially fatal outcome of DILD, patients receiving any antineoplastic drugs who have these predictors of a poor prognosis should be managed carefully throughout their treatments.

Among the HRCT imaging findings, the DAD pattern was an independent prognostic factor in severe DILD patients. The mortality rate was significantly higher in the group with the DAD pattern (DAD, 53.3\% vs. non-DAD, 13.3\%). Among the previous reports, only one observational study examining nivolumab-induced DILD reported the DAD pattern as a statistically significant predictor of a poor prognosis (Saito et al. 2020). The high mortality rate in patients with the DAD pattern agrees with the findings of previous reports on DILD (40-83.3\%) (Gemma et al. 2014, 2019, 2020; Shi et al. 2014; Hotta et al. 2010; Tomii et al. 2017). A propensity of certain drugs to cause DAD was not identified in our study. Nevertheless, this study reviewed and analyzed a sufficient number of severe DILD cases. The results emphasize that patients with the DAD pattern have a high mortality rate and a poor prognosis regardless of the type of cancer or antineoplastic drug treatment.

The present study had some limitations. First, admission criteria vary among physicians, and there is a possibility that the severity of DILD may not be consistent. CTCAE ver. 5. defines Grade 3 pneumonia as "Severe symptoms; limiting self-care ADL; oxygen indicated." More than $70 \%$ of patients in our patient population required oxygen 
demand, and all patients were receiving steroids. Additionally, since we excluded observational cases, our definition of "severe" DILD is somewhat similar to CTCAE grade 3 pneumonitis. Second, the study was performed retrospectively at a single institution. However, severe DILD is not frequently encountered in clinical practice, and the progress of multiple cases can be challenging to follow in detail. Nevertheless, our institution has a large caseload of patients requiring cancer medications. We reviewed the drug histories of all the patients who received antineoplastic drugs, and board-certified diagnostic radiologists reviewed the CT images of patients who developed severe DILD. Thus, the consecutiveness of cases is authentic, to some extent. Finally, we could not obtain pathological diagnoses in most of the cases. A previous report has described a concordance between HRCT interpretation and tissue diagnosis of about $50 \%$ in patients with DILD (Cleverley et al. 2002). This result supports the use of HRCT as a non-invasive test to predict prognosis, to some extent, when a bronchoscopic alveolar lavage or biopsy cannot be performed.

In conclusion, across all cancer types and all antineoplastic agents, the frequency of DILD was highest among patients with thoracic cancer. In addition, the DAD pattern, concurrent/previous ILD, and a PS of 2-4 were associated with a poor outcome. Therefore, from the perspective of DILD, a special attention should be paid to the use of antineoplastic agents in patients with thoracic cancer and other patients with the factors mentioned above.

Acknowledgements We would like to express our sincere appreciation to all the patients who participated in this study and to their families and the staff of the Department of Diagnostic Radiology, Department of Thoracic Oncology, Department of Medical Oncology, Department of Hepatobiliary and Pancreatic Oncology, Department of Gastrointestinal Medical Oncology, Department of Dermatologic Oncology, Department of Musculoskeletal Oncology, National Cancer Center Hospital, for their assistance, and especially the staff of the Department of Medical information for organizing and providing a massive amount of data. This research did not receive any specific grant from funding agencies in the public, commercial, or not-for-profit sectors.

Author contributions SK and $\mathrm{HH}$ made contributions to the conception and design, acquisition of data, and data analysis. SK drafted the manuscript. $\mathrm{HH}$ and MK made substantial contributions to the study design and revision of the manuscript. SK analyzed and interpreted data and edited the manuscript. All authors critically reviewed the manuscript, and all approved the final version submitted for publication.

Funding The authors declare that this study was not funded.

Availability of data and materials The datasets generated during the current study are not publicly available due to ethical restrictions, but are available from the corresponding author on reasonable request.

\section{Declarations}

Conflicts of interest Dr. Horinouchi received research grants from MSD K.K., Chugai Pharmaceutical Co., Ltd., Genomic Health, Inc., Ono Pharmaceutical Co. Ltd, Bristol-Myers Squibb, Daiichi Sankyo Company, Limited, AstraZeneca K.K, AbbVie GK, and received honoraria from Eli Lilly Japan K.K., AstraZeneca K.K, Taiho Pharmaceutical Co., Ltd., Chugai Pharmaceutical Co., Ltd., MSD K.K., Novartis Pharma K.K., Takeda Pharmaceutical Co. Ltd., Kyowa Kirin Co., Ltd., Bristol-Myers Squibb. Dr. Yonemori had advisory roles of Chugai Pharmaceutical Co., Ltd., Ono Pharmaceutical Co. Ltd., and received honoraria from Takeda Pharmaceutical Co. Ltd., AstraZeneca K.K, Pfizer Inc., Chugai Pharmaceutical Co., Ltd., Eisai Co., Ltd., Eli Lilly Japan K.K. Dr. Okusaka's advisory roles were Taiho Pharmaceutical Co., Ltd., Daiichi Sankyo Company, Limited, Sumitomo Dainippon Pharma Co., Ltd., Bristol-Myers Squibb, AstraZeneca K.K., Eisai Co., Ltd., Nihon Servier. In addition, Dr.Okusaka received honoraria from ElmediX, Meiji Seika Pharma Co., Ltd., MSD K.K., AstraZeneca K.K, AbbVie GK, Eisai Co., Ltd., Ono Pharmaceutical Co. Ltd, Yakult Honsha Co.,Ltd., Shire plc, Daiichi Sankyo Company, Limited, Taiho Pharmaceutical Co., Ltd., Takeda Pharmaceutical Co. Ltd., Chugai Pharmaceutical Co., Ltd., Teijin Pharma Limited., Eli Lilly Japan K.K., Nippon Shinyaku Co., Ltd., Nihon Servier, Novartis Pharma K.K., Bayer Yakuhin, Ltd., Pfizer Inc., Mundipharma K.K., and received research grants from AstraZeneca K.K., Eisai Co., Ltd., Bristol-Myers Squibb, MSD K.K., Sumitomo Dainippon Pharma Co., Ltd. Dr. Boku received honoraria for lecture fees from Ono Pharmaceutical Co. Ltd., and Taiho Pharmaceutical Co., Ltd., and a research grant from Ono Pharmaceutical Co. Ltd. and Takeda Pharmaceutical Co. Ltd. Dr.Yamazaki reports personal fees and non-financial support from Ono Pharmaceutical Co. Ltd., Bristol-Myers Squibb, Novartis Pharma K.K., MSD K.K., individual fees from Merck Serono Co., Ltd., Takeda Pharmaceutical Co. Ltd., Chugai Pharmaceutical Co., Ltd. Dr. Kawai has received honoraria for lecture fees from Taiho Pharmaceutical Co., Ltd., Eli Lilly Japan K.K., Eisai Co., Ltd., Takara Bio Inc., Daiichi Sankyo, Bayer Yakuhin, Ltd., Kyowa Kirin Co., Ltd., Novartis Pharma K.K., and research expenses from Taiho Pharmaceutical Co., Ltd., Eli Lilly Japan K.K., Eisai Co., Ltd., Takara Bio Inc., and Daiichi Sankyo Company, Limited. Dr. Ohe had the advisory role of AstraZeneca K.K., Chugai Pharmaceutical Co., Ltd., Ono Pharmaceutical Co. Ltd., Bristol-Myers Squibb, Kyorin Pharmaceutical Co., Ltd., Celltrion Pharm Inc., Amgen Inc., and Nippon Kayaku Co., Ltd. In addition, Dr.Ohe received personal fees from AstraZeneca K.K., Chugai Pharmaceutical Co., Ltd., Eli Lilly Japan K.K., Ono Pharmaceutical Co. Ltd, Bristol-Myers Squibb, Boehringer Ingelheim, Bayer Yakuhin, Ltd., Pfizer Inc., MSD K.K., T Taiho Pharmaceutical Co., Ltd., Nippon Kayaku Co., Ltd., Kyowa Hakko Kirin Co., Ltd., and received research grants from AstraZeneca K.K., Chugai Pharmaceutical Co., Ltd., Lilly, Ono Pharmaceutical Co. Ltd, Bristol-Myers Squibb, Kyorin Pharmaceutical Co., Ltd., Sumitomo Dainippon Pharma Co., Ltd., Pfizer Inc., Taiho Pharmaceutical Co., Ltd., Novartis K.K, Ignyta Inc., Takeda Pharmaceutical Co. Ltd., Kissei Pharmaceutical Co., Ltd., Daiichi Sankyo Company, Limited, Janssen Pharmaceutical K.K., Loxo Oncology. Dr. Kusumoto received honoraria for lecture fees from AstraZeneca K.K. and Daiichi Sankyo Company, Limited, and research grants from Canon medical systems corporation. The remaining authors declare no conflict of interest.

Ethics approval and consent to participate The Ethics Review Committee of the National Cancer Center Central Hospital approved this study protocol (Approval number: 2019-069).

Consent for publication This manuscript contains no individual data. 
Open Access This article is licensed under a Creative Commons Attribution 4.0 International License, which permits use, sharing, adaptation, distribution and reproduction in any medium or format, as long as you give appropriate credit to the original author(s) and the source, provide a link to the Creative Commons licence, and indicate if changes were made. The images or other third party material in this article are included in the article's Creative Commons licence, unless indicated otherwise in a credit line to the material. If material is not included in the article's Creative Commons licence and your intended use is not permitted by statutory regulation or exceeds the permitted use, you will need to obtain permission directly from the copyright holder. To view a copy of this licence, visit http://creativecommons.org/licenses/by/4.0/.

\section{References}

Abdel-Rahman O, Elhalawani H (2015) Risk of fatal pulmonary events in patients with advanced non-small-cell lung cancer treated with EGF receptor tyrosine kinase inhibitors: a comparative meta-analysis. Future Oncol 11(7):1109-1122. https://doi.org/10.2217/fon. 15.16

Ando M, Okamoto I, Yamamoto N, Takeda K, Tamura K, Seto T, Ariyoshi Y, Fukuoka M (2006) Predictive factors for interstitial lung disease, antitumor response, and survival in non-smallcell lung cancer patients treated with gefitinib. J Clin Oncol 24(16):2549-2556. https://doi.org/10.1200/JCO.2005.04.9866

Binder D, Hübner RH, Temmesfeld-Wollbrück B, Schlattmann P (2011) Pulmonary toxicity among cancer patients treated with a combination of docetaxel and gemcitabine: A meta-analysis of clinical trials. Cancer Chemother Pharmacol 68(6):1575-1583. https://doi.org/10.1007/s00280-011-1648-2

Bouros D, Hatzakis K, Labrakis H, Zeibecoglou K (2002) Association of malignancy with diseases causing interstitial pulmonary changes. Chest, vol 121. Elsevier, Amsterdam, pp 1278-1289

Cleverley JR, Screaton NJ, Hiorns MP, Flint JDA, Müller NL (2002) Drug-induced lung disease: high-resolution CT and histological findings. Clin Radiol 57(4):292-299. https://doi.org/10.1053/crad. 2001.0792

Dhokarh R, Li G, Schmickl CN, Kashyap R, Assudani J, Limper AH, Gajic O (2012) Drug-associated acute lung injury: a populationbased cohort study. Chest 142(4):845-850. https://doi.org/10. 1378/chest.11-2103

Fine JP, Gray RJ (1999) A proportional hazards model for the subdistribution of a competing risk. J Am Stat Assoc 94(446):496-509. https://doi.org/10.1080/01621459.1999.10474144

Fujimoto D, Kato R, Morimoto T, Shimizu R, Sato Y, Kogo M, Ito J, Teraoka S, Nagata K, Nakagawa A, Otsuka K, Tomii K (2016) Characteristics and prognostic impact of pneumonitis during systemic anticancer therapy in patients with advanced non-smallcell lung cancer. PLoS ONE 11(12):e0168465. https://doi.org/10. 1371/journal.pone.0168465

Gemma A, Kudoh S, Ando M, Ohe Y, Nakagawa K, Johkoh T, Yamazaki N, Arakawa H, Inoue Y, Ebina M, Kusumoto M, Kuwano K, Sakai F, Taniguchi H, Fukuda Y, Seki A, Ishii T, Fukuoka M (2014) Final safety and efficacy of erlotinib in the phase 4 POLARSTAR surveillance study of 10708 Japanese patients with non-small-cell lung cancer. Cancer Sci 105(12):1584-1590. https://doi.org/10.1111/cas.12550

Gemma A, Kusumoto M, Kurihara Y, Masuda N, Banno S, Endo Y, Houzawa H, Ueno N, Ohki E, Yoshimura A (2019) Interstitial lung disease onset and its risk factors in Japanese patients with ALK-positive NSCLC after treatment with crizotinib. J Thorac Oncol 14(4):672-682. https://doi.org/10.1016/j.jtho.2018.11.022

Gemma A, Kusumoto M, Sakai F, Endo M, Kato T, Saito Y, Baba T, Sata M, Yamaguchi O, Yabuki Y, Nogi Y, Jinushi M, Sakamoto
K, Sugeno M, Tamura R, Tokimoto T, Ohe Y (2020) Real-world evaluation of factors for interstitial lung disease incidence and radiologic characteristics in patients with EGFR T790M-positive NSCLC treated with osimertinib in Japan. J Thorac Oncol 15(12):1893-1906. https://doi.org/10.1016/j.jtho.2020.08.025

Hamada T, Yasunaga H, Nakai Y, Isayama H, Matsui H, Fushimi K, Koike K (2016) Interstitial lung disease associated with gemcitabine: a Japanese retrospective cohort study. Respirology 21(2):338-343. https://doi.org/10.1111/resp.12665

Hotta K, Kiura K, Takigawa N, Yoshioka H, Harita S, Kuyama S, Yonei T, Fujiwara K, Maeda T, Aoe K, Ueoka H, Kamei H, Umemura S, Moritaka T, Segawa Y, Kawai H, Bessho A, Kato $\mathrm{K}$, Tabata M, Tanimoto M (2010) Comparison of the incidence and pattern of interstitial lung disease during erlotinib and gefitinib treatment in Japanese patients with non-small cell lung cancer: The okayama lung cancer study group experience. J Thorac Oncol 5(2):179-184. https://doi.org/10.1097/JTO.0b013 e3181ca12e0

Johkoh T, Lee KS, Nishino M, Travis WD, Ryu JH, Lee HY, Ryerson CJ, Franquet T, Bankier AA, Brown KK, Goo JM, Kauczor H-U, Lynch DA, Nicholson AG, Richeldi L, Schaefer-Prokop CM, Verschakelen J, Raoof S, Rubin GD, Hatabu H (2021) Chest CT diagnosis and clinical management of drug-related pneumonitis in patients receiving molecular targeting agents and immune checkpoint inhibitors: a position paper from the Fleischner Society. Radiology. https://doi.org/10.1148/radiol.2021203427

Leger P, Limper AH, Maldonado F (2017) Pulmonary toxicities from conventional chemotherapy. Clinics in chest medicine, vol 38. W.B. Saunders, USA, pp 209-222

Minami-Shimmyo Y, Ohe Y, Yamamoto S, Sumi M, Nokihara H, Horinouchi H, Yamamoto N, Sekine I, Kubota K, Tamura T (2012) Risk factors for treatment-related death associated with chemotherapy and thoracic radiotherapy for lung cancer. J Thorac Oncol 7(1):177-182. https://doi.org/10.1097/JTO.0b013e3182 $3 \mathrm{c} 4 \mathrm{c} 07$

Nakagawa K, Kudoh S, Ohe Y, Johkoh T, Ando M, Yamazaki N, Seki A, Takemoto S, Fukuoka M (2012) Postmarketing surveillance study of erlotinib in Japanese patients with non-smallcell lung cancer (NSCLC): an interim analysis of 3488 patients (POLARSTAR). J Thorac Oncol 7(8):1296-1303. https://doi.org/ 10.1097/JTO.0b013e3182598abb

Nishino M, Giobbie-Hurder A, Hatabu H, Ramaiya NH, Hodi FS (2016) Incidence of programmed cell death 1 inhibitor-related pneumonitis in patients with advanced cancer: a systematic review and meta-analysis. JAMA Oncol 2(12):1607-1616. https://doi. org/10.1001/jamaoncol.2016.2453

Osawa M, Kudoh S, Sakai F, Endo M, Hamaguchi T, Ogino Y, Yoneoka M, Sakaguchi M, Nishimoto H, Gemma A (2015) Clinical features and risk factors of panitumumab-induced interstitial lung disease: a postmarketing all-case surveillance study. Int $\mathrm{J}$ Clin Oncol 20(6):1063-1071. https://doi.org/10.1007/s10147-015-0834-3

Qi WX, Sun YJ, Shen Z, Yao Y (2015) Risk of interstitial lung disease associated with EGFR-TKIs in advanced non-small-cell lung cancer: a meta-analysis of 24 phase III clinical trials. J Chemother 27(1):40-51. https://doi.org/10.1179/1973947814Y.0000000189

Saito Y, Sasaki S, Oikado K, Tominaga J, Sata M, Sakai F, Kato T, Iwasawa T, Kenmotsu H, Kusumoto M, Baba T, Endo M, Fujiwara Y, Sugiura H, Yanagawa N, Ito Y, Sakamoto T, Ohe Y, Kuwano K (2020) Radiographic features and poor prognostic factors of interstitial lung disease with nivolumab for non-small cell lung cancer. Cancer Sci. https://doi.org/10.1111/cas.14710

Sakurada T, Kakiuchi S, Tajima S, Horinouchi Y, Okada N, Nishisako H, Nakamura T, Teraoka K, Kawazoe K, Yanagawa H, Nishioka Y, Minakuchi K, Ishizawa K (2015) Characteristics of and risk factors for interstitial lung disease induced by chemotherapy for 
lung cancer. Ann Pharmacother 49(4):398-404. https://doi.org/ $10.1177 / 1060028014566446$

Shi L, Tang J, Tong L, Liu Z (2014) Risk of interstitial lung disease with gefitinib and erlotinib in advanced non-small cell lung cancer: a systematic review and meta-analysis of clinical trials. Lung Cancer 83(2):231-239. https://doi.org/10.1016/j.lungcan.2013. 11.016

Tirumani SH, Ramaiya NH, Keraliya A, Bailey ND, Ott PA, Hodi FS, Nishino M (2015) Radiographic profiling of immune-related adverse events in advanced melanoma patients treated with ipilimumab. Cancer Immunol Res 3(10):1185-1192. https://doi.org/ 10.1158/2326-6066.CIR-15-0102

Toh CK, Wong EH, Lim WT, Leong SS, Fong KW, Wee J, Tan EH (2004) The impact of smoking status on the behavior and survival outcome of patient with advanced non-small cell lung cancer: a retrospective analysis. Chest 126(6):1750-1756. https://doi.org/ 10.1378/chest.126.6.1750

Tomii K, Kato T, Takahashi M, Noma S, Kobashi Y, Enatsu S, Okubo S, Kobayashi N, Kudoh S (2017) Pemetrexed-related interstitial lung disease reported from post marketing surveillance (malignant pleural mesothelioma/non-small cell lung cancer). Jpn J Clin Oncol 47(4):350-356. https://doi.org/10.1093/jjco/hyx010

Publisher's Note Springer Nature remains neutral with regard to jurisdictional claims in published maps and institutional affiliations. 